\section{JTI}

JOURNAL OF

TRAUMA AND INJURY

\title{
Upper Extremity Deep Vein Thrombosis after Clavicle Fracture and Immobilization
}

Sung Jin Kim, M.D., Dae Sung Ma, M.D., Sung Youl Hyun, M.D., Yang Bin Jeon, M.D., Seok Joo, M.D. Ahram Han, M.D.

Department of Trauma Surgery, Gachon University Gil Medical Center, Incheon, Korea

Received: July 16, 2017

Revised: September 14, 2017

Accepted: October 25, 2017

\section{Correspondence to}

Dae Sung Ma, M.D.

Department of Trauma Surgery, Gachon University Gil Medical Center, 21 Namdong-daero 774beon-gil, Namdong-gu, Incheon 21565, Korea

Tel: $+82-32-460-3010$

Fax: +82-32-460-2372

E-mail: pegamu@gilhospital.com
Upper extremity deep vein thrombosis (DVT) is an unusual condition compared to lower extremity DVT, and it represents about $10 \%$ of all DVTs. We report a case of upper extremity DVT after clavicle fracture and immobilization.

Keywords: Deep vein thrombosis; Upper extremity; Anticoagulant

\section{INTRODUCTION}

Deep vein thrombosis occurs mostly in the lower limbs in trauma patients. And its most common cause is immobilization. Sometimes deep vein thrombosis can be occur in upper limb.

\section{CASE REPORT}

An 87-year-old man was transferred to our trauma center after a motorcycle accident. He presented with pain and distortion of the right chest wall and ipsilateral mid-clavicle area. Physical examination and radiographic evaluation revealed fracture of right first to seventh ribs with hemopneumothorax, fracture of mid-shaft of ipsilateral clavicle (Fig. 1), and hematoma (about $5 \mathrm{~cm} \times 10 \mathrm{~cm}$ sized) at elbow area. There was no neurologic or vascular problem distally. He underwent emergent closed thoracosotmy for right side hemopneunothorax and a velpeau bandage was applied to the shoulder for clavicle fracture. He had no previous surgical or medical history, except dementia. He was a non-smoker. After admission to the general ward, a sling was placed to immobilize the arm while waiting for surgical treatment. Preoperative transthoracic 

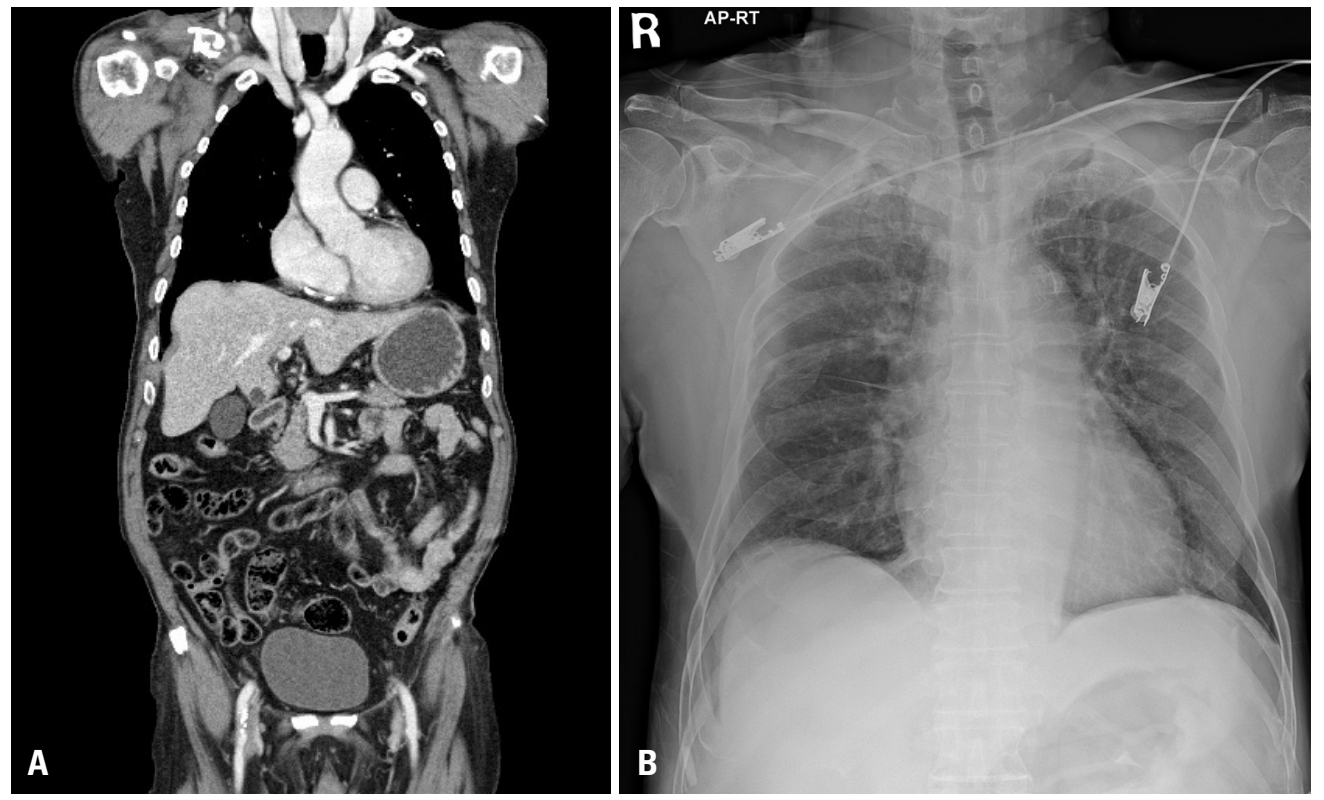

Fig. 1. Chest computed tomography and X-ray shows clavicle fracture (A) with multiple rib fracture (B).

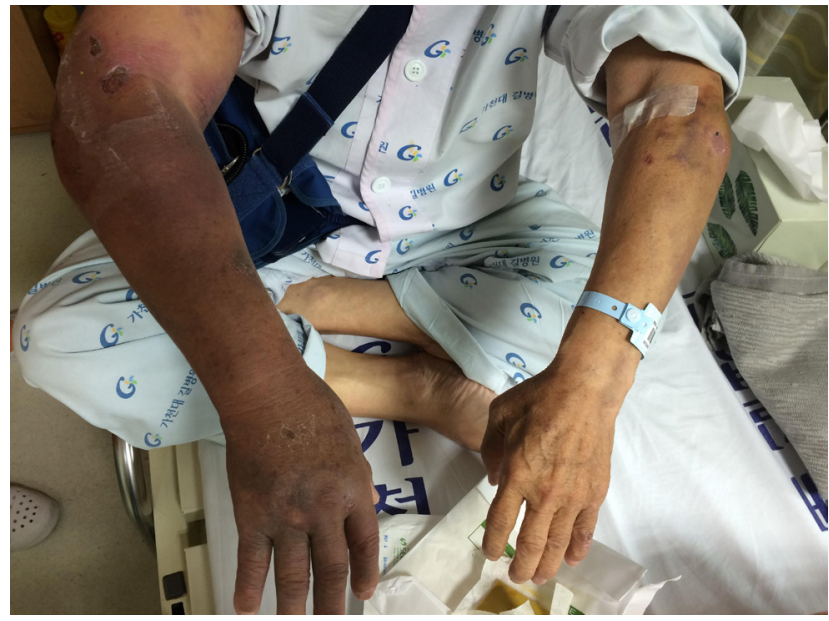

Fig. 2. Aggravated swelling and pain on right arm below axilla.

echocardiogram revealed normal cardiac function except relaxation abnormality of left ventricle filling pattern (early diastolic transmitral velocity to early myocardial velocity ratio $=10$ ). On 12th hospital day, the patient complained of aggravated swelling and pain on his right upper arm (Fig. 2). The laboratory examination showed increased serum D-dimer of $2.25 \mathrm{ug} / \mathrm{mL}$, which was slightly higher than the value five days before $(1.98 \mathrm{ug} / \mathrm{mL})$. Emergent brachial computed tomography (CT) angiography showed a deep vein thrombus (DVT) extending from cephalic vein to brachial vein along with hematoma and swelling of Rt. scalene muscle (Fig. 3). Anticoagulation therapy was started with enoxaparin $(1 \mathrm{mg} / \mathrm{kg}$ every 12 hours subcutaneous injection). After 6 days, the pain and swelling of the right arm and the axillary area were significantly reduced, but remnant partial thrombus was found at proximal brachial vein on Doppler ultrasonography. On hospital day 20, surgical operation was performed for mid-shaft clavicle fracture. The right clavicle was internally fixed with plate and wire after open reduction. After his clavicular surgery, anticoagulation was changed to warfarin with a target international normalized ratio of 1.5. He was discharged without further complication on 7 th postoperative day. At the 2-week follow-up, the patient had no complaints and the swelling of his upper arm had disappeared.

\section{DISCUSSION}

Upper extremity deep vein thrombosis (UEDVT) is an unusual condition compared to lower extremity DVT, and its incidence represents about $10 \%$ of all DVTs [1]. UEDVT is classified into primary and secondary according to its pathogenesis. Primary UEDVT is idiopathic thrombosis or those related to anatomical abnormalities. Secondary UEDVT represents $80 \%$ of all UEDVT cases. 


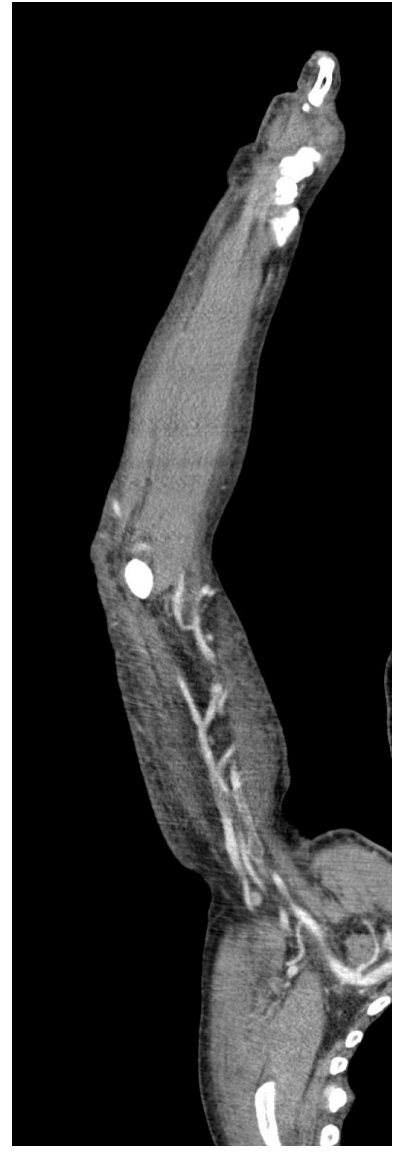

Fig. 3. Computed tomography angiography. The presence of a thrombus extending from right cephalic vein to brachial vein.

Secondary UEDVTs are those associated with various factors like malignancy, central venous catheter, oral contraceptative use etc. Trauma is reported to be responsible for $3-17 \%$ of secondary UEDVT [2].

Muñoz et al. [3] reported that UEDVT was less frequently associated with recent immobility compared to lower extremity deep vein thrombosis in their retrospective analysis of Registro Informatizado de Enfermedad TromboEmbolica (RIETE) registry. The authors also reported that only $9.0 \%$ of UEDVT had clinically overt pulmonary embolism (PE) compared to LEDVT (29\%). However, the incidence of major or fatal bleeding, recurrent DVT, recurrent PE or fatal PE of UEDVT were similar to that of LEDVT.

Mechanism of trauma related UEDVT is unclear. However, all three factors of the Virchow's triad of venous thrombosis [4]; the injuries in the vascular endothelium, alterations in normal blood flow, and hypercoagulability are thought to affect the development of UEDVT in trauma.
Clinical manifestations of UEDVT are edema, pain or erythema of the affected extremity. Our patient also complained of right upper arm swelling, redness, and pain. However, these findings are common in trauma patients with soft tissue injury and thus are nonspecific. Therefore, it is important to maintain a high index of suspicion in high-risk patients, and perform further evaluation when UEDVT is suspected. The diagnosis of UEDVT is not clearly standardized. Duplex ultrasonography has the advantage of being readily available and non-invasive, but when PE is suspected, CT venography or magnetic resonance venography is more useful. Classical venography is less frequently performed nowadays due to its invasiveness, however it still has an advantage when endovascular management for DVT is considered [4,5].

The 2008 American College of Chest Physicians' guidelines on venous thromboembolism recommends similar treatment for DVT in both upper and lower limbs. According to the guideline, UEDVT needs anticoagulation therapy with heparin, low molecular weight heparin or new oral anticoagulants upon diagnosis. Thrombolysis (systemic or trans-catheter) can provide a more rapid return of venous patency. Trans-catheter thrombolysis may be safer, but the research is yet limited. Thrombolysis catheter extraction and surgical thrombectomy is indicated in patients with large clots with high risk of embolization in patients who are absolutely contraindicated for thrombolysis and anticoagulation therapy [6].

Unlike LEDVT, UEDVT after trauma is known to be rare. Therefore, empiric or preventable anticoagulation therapy is not routinely recommended, and its diagnosis may be delayed without high index of suspicion. However, UEDVT can be potentially lethal when pulmonary embolism occurs. Trauma patients with upper extremity injury and subsequent immobilization of the injured extremity may benefit from medical prophylaxis. More research is needed to identify further risk factors of UEDVT and to clarify the risk-benefit of its prophylaxis.

\section{REFERENCES}

1. Bernardi E, Pesavento R, Prandoni P. Upper extremity deep venous thrombosis. Semin Thromb Hemost 2006;32:729-36. 
2. Spiezia L, Simioni P. Upper extremity deep vein thrombosis. Intern Emerg Med 2010;5:103-9.

3. Muñoz FJ, Mismetti P, Poggio R, Valle R, Barrón M, Guil $\mathrm{M}$, et al. Clinical outcome of patients with upper-extremity deep vein thrombosis: results from the RIETE Registry. Chest 2008;133:143-8.
4. Lee KA, Ramaswamy RS. Interventional approaches to deep venous thrombosis. Curr Opin Cardiol 2017;32:679-86.

5. Baarslag HJ, Koopman MM, Reekers JA, van Beek EJ. Diagnosis and management of deep vein thrombosis of the upper extremity: a review. Eur Radiol 2004;14:1263-74.

6. Dickson BC. Virchow's triad. Br J Haematol 2009;145:433. 